\title{
On the Essentials of Theoretical Education and Clinical Practice for
}

\section{Advanced Nursing}

\author{
Yanzhen Fan \\ Hospital of Qinzhou University \\ Qinzhou 535000, China \\ E-mail: angelfan_love@126.com
}

\begin{abstract}
The author of the present paper, on the basis of her current experience and deep practice, has suggested seven essentials of theoretical education and clinical practice for advanced nursing, which are centered and docomented the perspectives of the notions, talent training, responsibilities, development and forwardness of the advanced nursing further education and practice in the process of building up the harmonious society in China, while presented relevent strategies to ensure the program feasible of each essential.
\end{abstract}

Keywords: Essentials, Theoretical education, Clinical practice, Advanced nursing

\section{Introduction}

Nursing as a practice profession requires both practice experts and nurse scientists to expand the scientific basis for patient care. A series of theoretical education in nursing are designed to prepare nurses for the highest level of exercise and practice and scientific inquiry, which has great benefits for nurses specifically to prepare individuals for specialized nursing practice. Even more, individuals who acquire the the theoretical education and clinical practice for advanced nursing will seek to fill roles as educators and will use their considerable practice expertise to educate the next generation of nurses.

Some teaching strategies and learning principles will be incorporated into the curriculum for theoretical education and clinical practice for advanced nursing, as it relates to patient education. This preparation is in addition to that required for their area of specialized nursing practice or research in the case of the higher education.

\section{Essentials of Theoretical Education and Clinical Practice for Advanced Nursing}

The following essentials outline the curricular elements and competencies that must be present in programs conferring the theoretical education and clinical practice for advanced nursing, the trainees will be prepared for a variety of nursing practice roles (Zen Fengrui P29). The essentials delineated here address the foundational competencies that are core to all advanced nursing practice roles. However, the depth and focus of the core competencies will vary based on the particular role for which the trainee is preparing. For example, trainees preparing for organizational leadership or administrative roles will have increased depth in organizational and systems' leadership; those preparing for policy roles will have increased depth in health care policy; and those preparing for the roles such as nurse practitioners, clinical nurse specialists, nurse anesthetists, and nurse midwives, will have more specialized content in an area of advanced practice nursing.

Additionally, it is important to understand that the delineation of these competencies should not be interpreted to mean that a separate course for each of the essentials should be offered. Curricula will differ in emphases based on the particular specialties for which students are being prepared.

The essentials document outlines and defines the eight foundational essentials and provides some introductory comments on specialty competencies/content. The specialized content, as defined by specialty organizations, complements the areas of core content defined by the essentials and constitutes the major component of theoretical education and clinical practice for advanced nursing programs.

\subsection{Essential I: Scientific Underpinnings for Practice}

The practice in nursing provides the terminal academic preparation for nursing practice. The scientific underpinnings of 
this education reflect the complexity of practice at the doctoral level and the rich heritage that is the conceptual foundation of nursing. The discipline of nursing is focused on: The principles and laws that govern the life-process, well-being, and optimal function of human beings, sick or well; The patterning of human behavior in interaction with the environment in normal life events and critical life situations; the nursing actions or processes by which positive changes in health status are affected; and the wholeness or health of human beings recognizing that they are in continuous interaction with their environments (Donaldson \& Crowley, 1978). Preparation to address current and future practice issues requires a strong scientific foundation for practice. The scientific foundation of nursing practice has expanded and includes a focus on both the natural and social sciences. These sciences that provide a foundation for nursing practice include human biology, genomics, the science of therapeutics, the psychosocial sciences, as well as the science of complex organizational structures.

In addition, philosophical, ethical, and historical issues inherent in the development of science create a context for the application of the natural and social sciences. Nursing science also has created a significant body of knowledge to guide nursing practice and has expanded the scientific underpinnings of the discipline. Nursing science frames the development of middle range theories and concepts to guide nursing practice. Advances in the foundational and nursing sciences will occur continuously and nursing curricula must remain sensitive to emerging and new scientific findings to prepare theeducation and practice for advanced nursing for evolving clinical practice realities, which always carries on the goals to integrate nursing science with knowledge from ethics, the biophysical, psychosocial, analytical, and organizational sciences as the basis for the highest level of nursing practice, to use science-based theories and concepts to determine the nature and significance of health and health care delivery phenomena and health and health care delivery phenomena, and to develop and evaluate new practice approaches based on nursing theories and theories from other skills and clinical exercises.

\subsection{Essential II: Clinical Scholarship and Analytical Methods for Evidence-Based Practice}

Scholarship and research are the hallmarks of higher training education. Although basic research has been viewed as the first and most essential form of scholarly activity, an enlarged perspective of scholarship has emerged through alternative paradigms that involve more than discovery of new knowledge (Boyer, 1990). These paradigms recognize that (1) the scholarship of discovery and integration's reflects the investigative and synthesizing traditions of academic life.(Boyer, p. 21); (2) scholars give meaning to isolated facts and make connections across disciplines through the scholarship of integration; and (3) the scholar applies knowledge to solve a problem via the scholarship of application (referred to as the scholarship of practice in nursing). This application involves the translation of research into practice and the dissemination and integration of new knowledge, which are key activities of trainees of the theoretical education and clinical practice for advanced nursing. The scholarship of application expands the realm of knowledge beyond mere discovery and directs it toward humane ends. Nursing practice epitomizes the scholarship of application through its position where the sciences, human caring, and human needs meet and new understandings emerge.

Nurses have long recognized that scholarly nursing practice is characterized by the discovery of new phenomena and the application of new discoveries in increasingly complex practice situations, which is especially constructed and admitted in the nursing field of China. The integration of knowledge from diverse sources and across disciplines, and the application of knowledge to solve practice problems and improve health outcomes are only two of the many ways new phenomena and knowledge are generated other than through research. Research-focused doctoral programs in nursing are designed to prepare graduates with the research skills necessary for discovering new knowledge in the discipline. In contrast, the nurses trained with theoretical education and clinical practice for advanced nursing should engage more in advanced nursing practice, use analytic methods to critically appraise existing evidences to determine, implement the best facts for practice and population trends, evaluate quality improvement methodologies to promote safe, timely, effective, efficient, equitable, and patient-centered care, apply relevant findings to develop practice guidelines and improve practice and the practice environment. And disseminate findings from evidence-based practice and research to improve healthcare outcomes, which is vital for today China's public nursing care and health concern.

\subsection{Essential III: Information Systems and Patient Care Technology for the Improvement and Transformation of Health Care}

Those who are undergoing the theoretical education and clinical practice for advanced nursing are distinguished by their abilities to use information systems/technology to support and improve patient care and healthcare systems, and provide leadership within healthcare systems and/or academic settings. Knowledge and skills related to information systems/technology and patient care technology prepare them to apply new knowledge, manage individual and aggregate level information, and assess the efficacy of patient care technology appropriate to a specialized area of practice and care systems. Information systems provide a mechanism to apply budget and productivity tools, practice information systems and decision supports, and web-based learning or intervention tools to support and improve patient care. 


\subsection{Essential IV: Health Care Policy for Advocacy in Health Care}

Health care policy--whether it is created through governmental actions, local decision making, or organizational standards--creates a framework that can facilitate or impede the delivery of health care services or the ability of the provider to engage in practice to address health care needs. Thus, engagement in the process of policy development is central to creating a health care system that meets the needs of its constituents. Political activism and a commitment to policy development are central elements of professional nursing practice. Health policy influences multiple care delivery issues, including health disparities, cultural sensitivity, ethics, the internationalization of health care concerns, access to care, quality of care, health care financing, and issues of equity and social justice in the delivery of health care.

As we know, each individual in the advanced nursing has the capacity to engage proactively in the development and implementation of health policy at all levels, including institutional, local, state, regional, federal, and international levels. Preparing graduates with the essential competencies to assume a help and a need in the development of health policy requires that the nurses have opportunities to contrast the major contextual factors and policy triggers that influence health policy-making at the various levels, such as critically analyze health policy proposals, health policies, and related issues from the perspective of consumers, nursing, other health professions, and other stakeholders in policy and public forums; influence policy makers through active participation on committees, boards, or task forces at the institutional, local, state, regional, national, and/or international levels to improve health care delivery and outcomes; educate and set up exmaples for others; advocate for the nursing profession within the policy, healthcare communities, social justice, equity, and ethical policies within all healthcare areas.

\subsection{Essential V: Interprofessional Collaboration for Improving Patient and Population Health Outcomes}

Today's complex, multi-tiered health care environment in the urban and rural areas in China depends on the contributions of highly skilled and knowledgeable individuals from multiple professions. In order to accomplish the perfect policy "Public Medical Care and Cure" for safe, timely, effective, efficient, equitable, and patient-centered care in harmonious China, healthcare professionals must function as highly collaborative teams and be fluid depending upon the patients' needs. Therefore, nurses have preparation in methods of effective teamspirit with the doctors and are prepared to play a central role in establishing interprofessional teams, participating in the work of the team, and assuming cooperative collectives of the team when appropriate to realize the ideals of employing effective communication and collaborative skills in the development and implementation of practice models, peer review, practice guidelines, health policy, standards of care, and/or other scholarly products(Cheng Jiae P77).

\subsection{Essential VI: Clinical Prevention and Public Health for Improving the Nation's Health}

Clinical prevention is defined as health promotion and risk reduction/illness prevention for individuals and families. Population health is defined to include aggregate, community, environmental/occupational, and cultural/socioeconomic dimensions of health. Aggregates are groups of individuals defined by a shared characteristic such as gender, diagnosis, or age. These framing definitions are endorsed by representatives of multiple disciplines including nursing (Allan et al., 2004). The implementation of clinical prevention and population health activities is central to achieving the national goal of improving the health status of the population of China. In an effort to address this national goal, it appears very important ot support the transformation of clinical education by creating an objective to increase the proportion of schools of medicine, nursing, and other health professionals that have a basic curriculum that includes the core competencies in health promotion and disease prevention (Allan et al., 2004). Nurses trained in the theoretical education and clinical practice for advanced nursing would easily engage in leadership to integrate and institutionalize evidence-based clinical prevention and population health services for individuals, aggregates, and populations. Consistent with these national calls of building up a harmonious society for action and with the longstanding focus on health promotion and disease prevention in nursing curricula and roles, they have a foundation in clinical prevention and population health. This foundation will enable the trained nurses to analyze epidemiological, biostatistical, occupational, and environmental data in the development, implementation, and evaluation of clinical prevention and population health.

\subsection{Essential VII: Advanced Nursing Practice}

The increased knowledge and sophistication of healthcare has resulted in the growth of specialization in nursing in order to ensure competence in these highly complex areas of practice. The reality of the growth of specialization in nursing practice is that no individual can master all advanced roles and the requisite knowledge for enacting these roles. Theoretical education and clinical practice for advanced nursing surely provide preparation within distinct specialties that require expertise, advanced knowledge, and mastery in one area of nursing practice (Huang Jinyue P16). The relative nurse is prepared to practice in an area of specialization within the larger domain of nursing. Indeed, this distinctive specialization is a hallmark of the trained nurses. Such programs provide learning experiences that are based in a variety of patient care settings, such as hospitals, long-term care settings, home health, and/or community settings. These learning experiences should be integrated throughout the program of study, to provide additional practice 
experiences beyond those acquired in a baccalaureate nursing program. These experiential opportunities should be sufficient to inform practice decisions and understand the patient care consequences of decisions. Because a variety of differentiated roles and positions may be held by the trainees, role preparation for specialty nursing practice, including legal and regulatory issues, is part of every program's curricula, such as how to conduct a comprehensive and systematic assessment of health and illness parameters in complex situations, incorporating diverse and culturally sensitive approaches; how to design, implement, and evaluate therapeutic interventions based on nursing science and other sciences; how to develop and sustain therapeutic relationships and partnerships with patients (individual, family or group) and other professionals to facilitate optimal care and patient outcomes; and how to demonstrate advanced levels of clinical judgment, systems thinking, and fiscal issues.

\section{Conclussion}

Analyizing the essentials of theoretical education and clinical practice just presentign the focus on advanced nursing practice, on expanding responsibility and accountability in the care and management of individuals and families, and on carrying on the socialist medical spirits in the harmonious construction of China. By virtue of this direct care focus, the nurses, developing additional competencies in direct practice and in the guidance and coaching of individuals and families through developmental, health-illness, and situational transitions, working with diverse stakeholders for interor intra-organizational achievement of health-related organizational or public policy goals; and, designing patient-centered care delivery systems or policy level delivery models, must bring about great efforts and benefits for modern China.

\section{References}

Allan, J., Agar Barwick, T., Cashman, S., Cawley, J. F., Day, C., Douglass, C. W., et al. (2004). Clinical prevention and population health: Curriculum framework for health professions. American Journal of Preventive Medicine, 27(5), 471-476.

Boyer, E. L. (1990). Scholarship reconsidered: Priorities of the professoriate. Princeton, NJ: Carnegie Foundation for the Advancement of Teaching.

Cheng Jiae. (2006). Talking about how to train advanced nursing talent. Health Vocational Education, 24(13).

China Association of Colleges of Nursing. (2001). The essentials of baccalaureate education for professional nursing practice. Beijing: Publishing House of Chinese Medicine.

Donaldson, S., \& Crowley, D. (1978). The discipline of nursing. Nursing Outlook, 26(2), 113-120.

Huang, Jinyue. (2008). Introduction to Advanced Nursing Practice. Beijing: Pubishing House of People's Health.

Zen, Fengrui. (2005). Advanced practice nursing: An integrative approach. Medicine and Nursing in China, (2). 